\title{
ROSARIO VERA PEÑALOZA UNA MAESTRA QUE DEJÓ HUELLA EN LA HISTORIA DE LA EDUCACIÓN DE LA ARGENTINA ${ }^{1}$
}

\author{
María Cristina Vera de Flachs ${ }^{2}$ \\ CONICET- Universidad Nacional de Córdoba - Argentina \\ Grupo de Investigación HISULA \\ vera@onenet.com.ar
}

Recepción: 27/01/2012

Evaluación: $15 / 02 / 2012$

Aceptación: 29/05/2012

Artículo de Reflexión

doi: 10,9757

\section{RESUMEN}

El objetivo de este trabajo es repensar la vida y obra de Rosario Vera Peñaloza, una maestra normalista que a lo largo de su existencia ha sido sin duda un referente en el proceso educativo argentino. Para realizar la tarea nos hemos

\section{ROSARIO VERA PEÑALOZA AN EDUCATOR THAT SWAYED INTO ARGENTINEAN TEACHING HISTORY}

\section{ABSTRACT}

This article's rationale aims to reconsider Rosario Vera Peñaloza's lifetime and work. A former educator who has undoubted been a main reference in Argentinean teaching process through her existence. To fulfill this task we had analyzed different authors 'devoted work about her as well as her own master pieces.

Key words: Journal of Latin American Education History, Argentina, new schooling, 20th century, first museum in Argentina, kindergarten. valido de su propia obra y de los autores que se dedicaron a estudiarla.

Palabras clave: Revista Historia de la Educación Latinoamericana, Argentina, escuela nueva, siglo XX, primer museo argentino, jardines de infantes.

\section{ROSARIO VERA PEÑALOZA UMA PROFESSORA QUE DEIXOU SUA MARCA NA HISTÓRIA DA EDUCAÇÃO ARGENTINA}

\section{RESUMO}

O objetivo deste trabalho é repensar a vida e obra de Rosario Vera Peñaloza, uma professora normalista que ao largo de sua existência foi sem dúvida uma referencia no processo educativo argentino. Para realizar esta tarefa nos valemos de sua própria obra e de outros autores que se dedicaram a estudá-la.

Palavras - chave: Revista História da Educação Latino-americana, Argentina, escola nova, século XX, primeiro museu argentino, jardins de infância.

1 Nos ocupamos de esta maestra en el artículo titulado "Rosario Vera Peñaloza: maestra de la patria" en Diana Soto Arango et. al., Educadores latinoamericanos, T. IV, Siglos XX y XXI. (Tunja: Ediciones Doce Calles S.L., 2011), 17.

2 Doctora en Historia, Licenciada en Historia por la Facultad de Filosofia y Humanidades, profesora titular de historia social contemporánea de la Universidad Nacional de Córdoba, investigadora principal del Consejo Nacional de la Investigaciones Científicas y Tecnológicas [CONICET] e integrante del grupo de investigación Historia y Prospectiva de la Universidad Latinoamericana - HISULA, adscrito a la Universidad Pedagógica y Tecnológica de Colombia. Artículo vinculado al proyecto de investigación la Universidad en la capacitación de maestras rurales en Colombia y Guatemala SGI 1034 y proyecto Historias de vida. Maestras rurales de mediados del siglo XX y XXI en la zona cundiboyacense SGI 980 del grupo de investigación HISULA - UPTC. 


\section{INTRODUCCIÓN}

El género biográfico es un método revalorado en la historiografía argentina desde los años sesenta del siglo XX habiendo, en estas últimas décadas, avanzado notablemente gracias a la cantidad de estudios realizados. Las historias de vida incluyen la descripción de las experiencias y acontecimientos más significativos de la vida del sujeto a estudiar, para a través de ellas dar cuenta de los sentimientos, modos de ver y concebir el mundo que los rodea. Esto debe necesariamente llevarnos a indagar la vida y obra de Rosario Vera Peñaloza pero, a su vez, dar cuenta de sus relaciones y las estrategias que formularon los distintos actores que compartieron con ella las distintas etapas de su existencia. Hacemos nuestras las palabras que, en 1842, decía Domingo F. Sarmiento sobre el tema, porque sin duda resume nuestras intenciones cuando nos propusimos este trabajo:

"La biografía de un hombre que ha desempeñado un gran papel en una época y un país dados, es el resumen de la historia contemporánea, iluminada con los animados colores que reflejan las costumbres y bábitos nacionales, las ideas dominantes, las tendencias de la civilización, y la dirección especial que el genio de los grandes hombres puede imprimir a la sociedad... en su vida privada, en su carácter especial y en las doctrinas en que habian sido educados, se encuentra más bien la explicación de sus hechos públicos que no en las narraciones simplemente bistóricas...””.

Basándonos en estas premisas emprendemos la tarea intentando una aproximación biográfico-narrativa sobre la vida de esta maestra luchadora hasta el fin de su vida.

\section{La educación Argentina desde los inicios de la modernidad a la primera mitad del siglo $\mathrm{XX}$}

El primer Censo Nacional de Población levantado en la República Argentina en 1869 reveló que el total de población ascendía a 1.737.076 habitantes de los cuales más de un millón no sabían leer ni escribir. Esta realidad disparó los sueños de Domingo Faustino Sarmiento, quien, durante su exilio y después de visitar los pueblos más avanzados del orbe, imaginó implantar en su país un moderno sistema que expandiera la educación pública y permitiera superar aquellos guarismos.

Domingo F. Sarmiento, “De las biografías, El Mercurio 20/3/1842", en Polémica literaria, (Buenos Aires: Cartago, 1955), 30. 
Después de la segunda mitad del siglo XIX las élites dirigentes apoyándose en las recetas del liberalismo estaban convencidas que Argentina ocuparía un lugar importante en el mundo. $\mathrm{Y}$ es Sarmiento quien continuará la senda trazada por Bartolomé Mitre haciendo avanzar a la República "en una vía donde todo lo que ella puede producir y dar (gracias a sus riquezas naturales, a su posición en América y al genio civilizador de sus hijos), será obtenido bajo la égida de leyes respetadas de una completa seguridad"'. En este contexto, la ciencia y la educación laica serán claves para entrar a la modernidad.

"La escuela! He aqui el punto donde deben dirigirse las miradas de todos los ciudadanos inteligentes, para cambiar nuestras masas ignorantes en ciudadanos conocedores de sus derechos y sus deberes sociales..." ".

Es que una sociedad progresista debía desterrar el analfabetismo, considerado como uno de los males sociales. Obviamente la educación de las mujeres será un tema de discusión y entre tantos de los que participaron de esos debates debe destacarse también a Sarmiento quien tempranamente escribió sobre el derecho de éstas a la educación inspirándose en lo que había podido observar en sus continuos viajes, particularmente en los Estados Unidos, país que vivía un clima de reivindicaciones femeninas. El 8 de octubre de 1875 sostuvo:

"La costura, el bordado y otros trabajos que ganaba para subsistir no le bastan abora ni para comer", por eso pensaba que era necesario que se abrieran escuelas normales de mujeres. De esa manera podian enseñar porque esa era su función natural y además porque el Estado economizaba en sueldos ya que ellas cobraban la mitad de los hombres porque "sus necesidades eran menores".

Sin embargo, la realidad era que unas pocas llegaban a tener nociones de lectura o escritura. El 11 de noviembre de 1876, después de inaugurar el tramo de la sección norte del Ferrocarril Central Córdoba a Tucumán Sarmiento visitó Córdoba y, en esa ocasión, asistió a una conferencia en el salón de actos de la Universidad a cuyo término se lo invitó a disertar y ante un auditorio bien heterogéneo, donde había una buena cantidad de público femenino, llegó a expresar que no estaba lejos el "día que [las niñas] asistan con sus libritos debajo del brazo a los cursos universitarios" 7 . Y así fue.

\footnotetext{
"El Eco de Córdoba", periódico de Córdoba, 27 de mayo de 1869, ํo 1877.

"El Eco de Córdoba", periódico de Córdoba, 23 de febrero de 1872, № 2672.

Domingo F. Sarmiento, "Discursos parlamentarios" en: Obras completas, T. XX, Vol., III, (Berlín: Sarmiento Editor, La Facultad, 1914).

7 María Cristina Vera de Flachs, De Comadronas a Obstetras. La institucionalización del saber-1a ed. (Córdoba: Báez Ediciones, 2011).
} 
A través de estas manifestaciones y de la literatura que iba dirigida a ellas se fue construyendo un modelo de identidad femenina que se iba distanciando poco a poco de las pautas tradicionales que imponían ser buena esposa y madre. Simultáneamente el país exhibía un proceso de cambio que se intensificó con los años: estaba ordenado constitucionalmente, con fronteras internas y externas sin problemas, abierto a una masiva corriente inmigratoria, a la modernización y al progreso. El positivismo era aceptado por vastos sectores de la intelectualidad, que se asumía como comtiana, spenceriana o darwinista.

La creación de las Escuelas Normales fue una experiencia positiva y exitosa para las mujeres que deseaban dedicarse a la docencia y para las más osadas que ambicionaban incursionar en las universidades, recintos hasta entonces vedadas a ellas. Entre 1870 y 1896 se establecieron en el país 38 Escuelas Normales según el modelo de la Escuela Normal de Paraná, muchas de ellas regentadas por las 65 maestras normalistas que llegaron desde Estados Unidos $^{8}$. Por su condición de extranjeras y protestantes no resultó fácil la presencia de esas mujeres. Córdoba fue una de las provincias que con mayor virulencia el catolicismo las combatió. Un periódico cordobés sostenía por esos días lo siguiente:

"Se descatoliza a la Nación y no tenemos una sola palabra para defender los sagrados derechos de la conciencia. Si queremos asegurar el porvenir de la República debemos conservar el carácter religioso de la enseñanza levantando la Nación entera contra la escuela laica, que se quiere imponernos en odio a las creencias católicas"'.

En ese ámbito de confrontación en 1884, el Congreso de la Nación debatió y aprobó la ley 1420 de educación común, gratuita y obligatoria ${ }^{10}$. A los diez años de su aplicación, el índice nacional de analfabetismo había

Con la creación en 1870 de la Escuela Normal de Paraná y dos Escuelas Normales, en 1874 en Buenos Aires, se inauguró la formación de maestros en el país. De orientación espiritualista al comienzo estuvo inspirada en las ideas del filósofo alemán Karl Krause, llegó a transformarse en positivismo pedagógico a posteriori (con Carlos Octavio Bunge, José María Ramos Mejía, Víctor Mercante, Alfredo Ferreyra y José Ingenieros, como referentes). Luis Alberto Flores, Rosario Vera Peñaloza. Su Vida y sus Pensamientos.

9 ElEco de Córdoba, periódico cordobés, 29 de julio de 1874.

10 La discusión acerca de la ley 1420 sancionada durante la presidencia de Julio A. Roca produjo uno de los debates más intensos y de largo alcance en la historia de la Educación Argentina. Distintos puntos de vista en torno a la enseñanza religiosa, a la escuela mixta y al control del Estado sobre la educación dividieron a los hombres de la generación del '80. Los liberales impulsaron un programa secularizador, por el cual la Iglesia Católica perdió parte de sus potestades en cuanto al registro civil, la educación y el matrimonio. En ese marco, la religión en las escuelas fue el nudo del debate. Finalmente, la ley aprobada no hizo mención al carácter laico de la educación pero la instrucción religiosa quedó en calidad de optativa, con autorización de los padres, y dictada fuera del horario escolar. 
descendido al 53,5\%; en 1914 se hallaba en el 35\% y, en 1938, en un 12\%. El progreso era notable y los esfuerzos realizados permitieron escolarizar a la mayoría de la población entre los 6 y 13 años pertenecientes a todos los sectores sociales ${ }^{11}$.

Después del golpe militar de junio de 1943 hubo un cambio radical en las ideas aunque pronto Juan Domingo Perón plantearía su preocupación por la política educativa. Las tendencias de tinte nacionalista intentarán eliminar las influencias foráneas y desde entonces el nacionalismo y el catolicismo más la política estuvieron presentes en los hombres y mujeres que protagonizaron los cambios de los años cuarenta. Un aspecto positivo de la gestión fue el incentivo a la escuela pre-escolar, la educación de los adultos obreros y la instalación de las escuelas fábricas. En ese contexto Rosario Vera Peñaloza se formó y actuó desde la última década del siglo XIX hasta 1950.

\section{Cuna riojana}

Rosario Vera Peñaloza nació el 25 de diciembre de 1873 en Atiles, Malanzán, un pueblito perdido de los Llanos riojanos en el hogar conformado por Don Eloy Vera y Mercedes Peñaloza, siendo la cuarta hija.

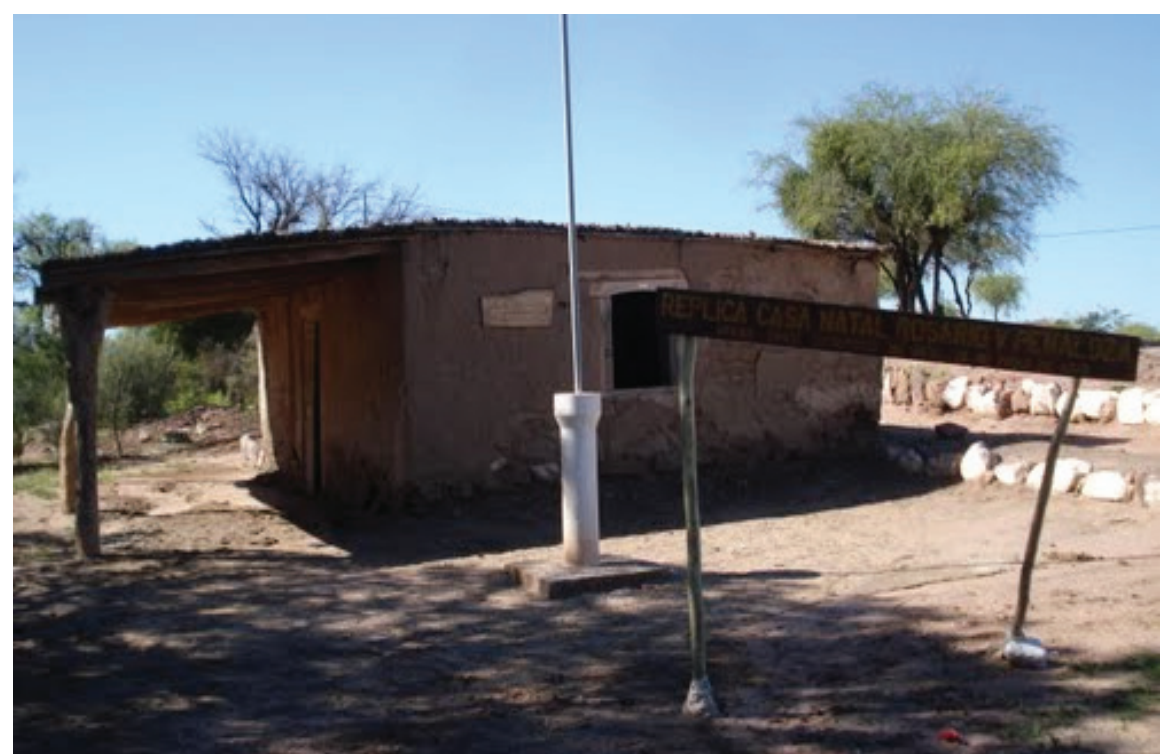

Casa natal de Rosário Vera Peñaloza.

$\overline{11}$ Bunge Alejandro E., en un libro de gran repercusión editado en 1940 titulado Una Nueva Argentina destacó el éxito de la lucha contra el analfabetismo. Capítulo XIX, "La Educación Argentina", Buenos Aires, Hyspamérica, 1984, p. 445 y ss. 
Descendiente de una familia de pura estirpe riojana vinculada a la historia civil y militar de esa provincia norteña quedó huérfana muy pequeña. En ese ambiente pueblerino y en compañía de su tía y madre de crianza doña Jesusa Peñaloza de Ocampo vio transcurrir sus primeros años de vida. A temprana edad fue llevada a San Juan, donde aprendió las primeras letras y concluyó la escuela primaria con Mary Graham, una de las maestras convocadas por Sarmiento ${ }^{12}$.

En el año 1884, Rosario regresó a La Rioja, su provincia natal e ingresó en la Escuela Normal, de donde egresó en 1888 con el título de maestra normalista. Como hemos señalado anteriormente la profesionalización de la enseñanza y la consiguiente mejora de la educación permitía a las jóvenes que no provenían de familias de altos ingresos una fuente de trabajo que les facultaba un medio de vida digna y una vía legítima de ascenso social.

\section{Su labor docente}

"Es asi como trabajamos aunque parez̧ca que jugamos..."

Pronto sus inquietudes y una gran vocación docente la llevaron a trasladarse a Paraná, donde en su Escuela Normal de Profesores obtuvo el título Superior de Enseñanza en 1894. Al año siguiente comenzó a ejercer como maestra de escuela primaria en la Escuela Modelo, particular dirigida por Felisa Latallada y en la Escuela Normal de Profesores. Ese itinerario lo hizo cargada de "Un puñado de ideales y otro de normas morales; he ahí el haber con que los riojanos salimos a rodar tierra; dos valores para las luchas más arduas son las que llevamos del solar nativo" ${ }^{13}$.

12 Mary Olstine Graham descendiente de escoceses, nació en 1842 en St. Louis Missouri, Estados Unidos, donde se graduó como maestra normalista especializada en Kindergarten en 1867. Contratada por Sarmiento llegó a Buenos Aires en 1879 y fue destinada a la Escuela Normal de Paraná. Poco después pasó a ejercer en la provincia de San Juan, donde se hizo cargo de la dirección de la Escuela Normal que había sido creada ese año. Tenía sólo 27 años, sin embargo eso no fue un obstáculo para que Sarmiento admirara su capacidad, lo que lo llevó a expresar que "había valido la pena esperar tanto". "Miss Mary" causó asombro en San Juan con la puesta en práctica de su método de enseñanza, basado en la observación y la experiencia. Su objetivo era inducir a los alumnos a analizar y comprender más que a memorizar. En 1883 llegaron a la provincia cuatro nuevas maestras norteamericanas: Clara Gillies, que venía a ocupar la vice dirección; Sarah y Florence Atkinson y Sarah Harrison. Cuando, al cabo de seis años, Mary Graham cumplió su segundo contrato, regresó a Estados Unidos, pero el pueblo sanjuanino preocupado por la posibilidad de perderla, le hizo prometer que volvería. Así lo hizo e incluso regresó con su hermana Martha y su cuñado. En 1887 fue nombrada para organizar y dirigir la Escuela Normal de La Plata, tarea que terminó en 1888, falleciendo en esa ciudad el 10 de marzo de 1902.

13 Mabel Brizuela, "La vida como magisterio" en Número Homenaje a Rosario Vera Peñaloza, (Córdoba: 1980). 3. 
Paralelamente deseando trabajar con los más pequeños, Rosario cursó el Profesorado en Kindergarten dirigido por Sara Chamberlain de Eccleston, aunque no hay constancia si se recibió.

Sara Chamberlain nació el 8 de Abril de 1840 en Lewisburg, Filadelfia, Estados Unidos. Fue enfermera en el ejército durante la Guerra Civil y allí conoció al capitán Charles Friederick Eccleston con quien casó el 24 de Octubre de 1866 y tuvo dos hijos, John -nacido en 1868 y Emily en 1869. Al fallecer su esposo en 1875 comenzó a prepararse para el nuevo campo de la enseñanza que surgía en esa época: el kindergarten (jardín de infantes). Cursó en la Escuela Normal de Ruth Burritts. En 1877 conoció a Domingo F. Sarmiento a través de la amistad de éste con las hermanas Elizabeth y Mary Peabody. Esta última había sugerido a Sarmiento la necesidad de implementar el kindergarten "como sustento de la educación primaria", y sería Sara la encargada de desarrollar con éxito la idea en la Argentina años después.

Para fines del siglo XIX habían egresado de él más de una decena de profesoras las que, en su mayoría, regresaron a sus provincias de origen para instalar los primeros jardines de infantes ${ }^{14}$. Una de ellas fue Rosario quien en 1898 regresó a La Rioja, donde en 1900 fundó el jardín de Infantes anexo a la Escuela Normal, primero de la provincia y que hoy lleva su nombre. Además se desempeñó como profesora de castellano y trabajo manual en virtud que además

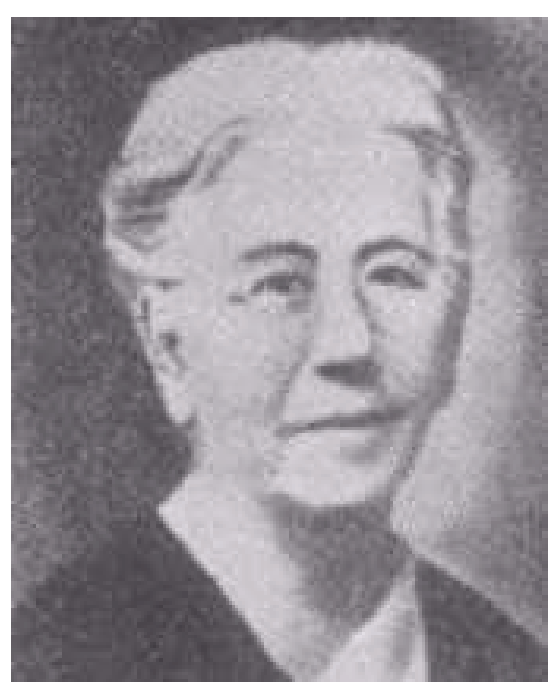

Rosario Vera Peñaloza (1873-1950) poseía títulos y conocimientos de Ejercicios Físicos, Dibujo, Trabajos manuales, Artes decorativas, Corte y confección, Grabado, Tejidos en telares, Modelado, etc. A partir de entonces esta docente se preocupó no sólo por emplear las técnicas aprendidas en su proceso de formación sino por aplicar dos móviles que estuvieron presentes a lo largo de su vida: el amor a la patria y la perfección del individuo. Años más tarde recordó ese momento con la siguiente frase: "...vuelvo al seno de mi Rioja amada, con el inmenso cariño que todos los hijos llevamos arraigados en el corazón..." $"$.

\footnotetext{
14 Rosario Vera Peñaloza, Custodia Zuloaga, Pía Didoménico y Rita Latallada de Victoria fueron las primeras maestras que fundaron jardines de infantes en diferentes puntos del país.

15 Instituto Social de la Universidad Nacional del Litoral, Biblioteca pedagógica, Pensamiento Vivo de Rosario Vera Peñaloza, (Universidad Nacional del Litoral: 1954).
} 
Dedicó su vida a la enseñanza. Fundó el primer jardín de infantes de su provincia natal y el primer museo para la escuela primaria. Fue inspectora de enseñanza secundaria, normal y especial. Falleció el 28 de mayo de 1950, jornada en la que se conmemora - en su honor- el Día de la Maestra Jardinera y el Día de los Jardines Maternales.

En 1906 decidió trasladarse a la ciudad de Córdoba para hacerse cargo de la vice dirección de la Escuela Normal de Córdoba donde al año siguiente se desempeñó como directora y como docente de Pedagogía y Matemáticas. La vida en esta ciudad le fue difícil hasta verse obligada a presentar su renuncia por mezquinos intereses políticos.

En 1911 como un gesto reparador las autoridades cordobesas la designan inspectora de Educación física y luego directora de la Escuela Normal Provincial Alberdi de esa ciudad, donde fundó un Jardín de Infantes.

Pero su labor sufrió otro traspié al ser separada de sus cargos, lo que la decide trasladarse a la Capital Federal. Tiempo después recordó esa instancia de la siguiente manera: "La lucha ha sido ardua, el camino difícil. A cada paso un obstáculo que debía sortear sin dejar aquel sagrado deber de fe, de esperanzas, de consagración, de dignidad para poder llevar con honor el nombre de riojana" ${ }^{16}$.

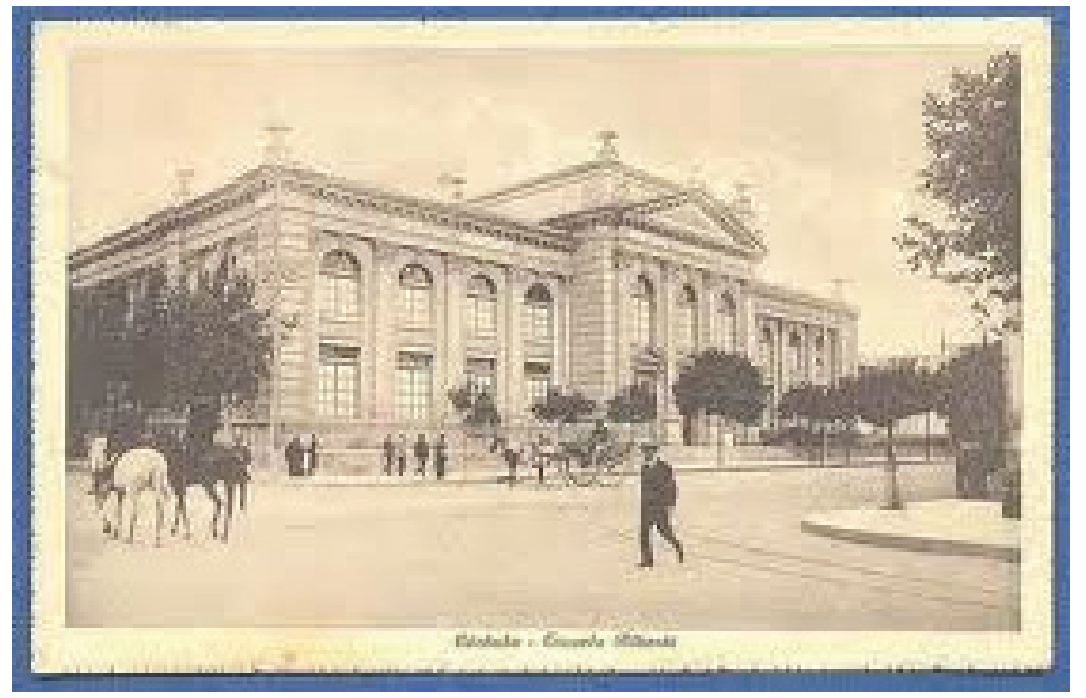

Foto 2.

Escuela

Alberdi

En ese ámbito entre 1912 y 1917 Rosario fue directora fundadora de la Escuela Normal "Roque Sáenz Peña" de la Capital Federal y directora fundadora de la Escuela Normal N 9 "Domingo Faustino Sarmiento".

16 Instituto Social de la Universidad Nacional del Litoral. 
Cuando tomó la dirección de la primera de las escuelas mencionadas tenía una matrícula de 227 alumnas el Normal y 300 en el Curso de Aplicación. Al dejar el cargo, la misma contaba con más de 1.500 alumnas. Es en esta etapa cuando en junio de 1915, elaboró un estudio comparativo de los sistemas Froebeliano y Montessori, recientemente implementado en una de las salas del Jardín Anexo a dicha Escuela.

\section{La Escuela Argentina modelo y sus últimos años de actividad}

En 1917 durante el gobierno de Hipólito Yrigoyen fue dejada cesante y Carlos María Biedma ${ }^{17}$ reconociendo sus valores le propone que colabore en la creación de la Escuela Argentina Modelo que se inauguró el 10 de abril de 1918, poniendo especial énfasis en la educación primaria. Con suma sencillez y modestia, Rosario sustituía al profesor que faltaba y más de una vez a los especialistas en Ciencias o Letras, con la ventaja de desempeñarse siempre como eximia pedagoga. Como una manera de reparar el agravio cometido en 1917, en 1924 otro gobernante radical Marcelo T. de Alvear le ofreció el cargo de inspectora de enseñanza secundaria, normal y especial, funciones que ejerció hasta 1926 cuando se retiró por razones de salud para acogerse a los beneficios de la jubilación.

Activa difusora de la cultura y animadora de grandes ideales, Rosario no dio por terminado su apostolado después de su jubilación sino que, por el contrario, fue entonces cuando comenzó a viajar por todo el país con el fin de asesorar a funcionarios, vecinos y maestros sobre los problemas de educación y cultura. Elaboró planes y programas de estudios para la escuela primaria y preescolar y dictó cursos especiales de perfeccionamiento que tuvieron reconocimiento oficial ${ }^{18}$, fundó bibliotecas y asistió a congresos pedagógicos, temas que desarrollaremos a continuación.

Su objetivo principal era impulsar la enseñanza popular que nos legara Sarmiento en tanto ella se enrolaba dentro de la corriente de los maestros democráticos- radicalizados, socialistas y anarquistas que cuestionaban la

17 Biedma nació el 1 de marzo de 1878. Fue alumno y luego profesor del Colegio Nacional de Buenos Aires, se doctoró en Derecho y Ciencias Sociales en la Universidad de Buenos Aires. Para su realización se inspiró en dos fuentes: la conducta religiosa y el amor a la patria, falleció el 9 de noviembre de 1946. Su idea está definida en el ideario de la Escuela: "Servir a la Nación en la tarea de educar con esmero, austeridad y valor a sus futuros ciudadanos". Tras su fallecimiento sus hijos Carlos José y Juan Martín aseguraron la continuidad del proyecto educativo; hoy sus nietos asumen el mensaje fundacional como propio.

18 Por ejemplo en setiembre de 1935 dictó un curso en la Escuela Normal Alejandro Carbó de la ciudad de Córdoba titulada "Como podemos hacer patria". 
burocratización del sistema y se interesaban por las ideas innovadoras. Este ideario los reunió y constituyeron las así llamadas Sociedades Populares de Educación, destacándose junto a Rosario, entre otros Carlos Vergara y Elvira Rawson de Dellepiane y Alicia Moreau de Justo ${ }^{19}$. Los propulsores de este movimiento popular a favor de la escuela y de la cultura incluían la "fiscalización de la labor docente", como una medida tendiente a quebrar el aislamiento en que vivía la escuela pública, en la que padres y maestros sólo se veían durante el curso lectivo cuando había que exponer alguna queja. Con el crecimiento en número y en impacto de las Sociedades de Educación Popular, surgió la necesidad de coordinar acciones y en 1909 se organizó el Primer Congreso de estas instituciones auspiciado por la Asociación Nacional del Profesorado, al que asistieron destacados educadores de todo el país que representaban a setenta y dos entidades ${ }^{20}$. Estos Congresos se repitieron en 1915, 1921 y 1930; este último marcó un hito por los temas tratados pues, entre otras cuestiones, se debatió sobre el analfabetismo, la educación de los adultos, la previsión social, la situación del magisterio, lo que llevó al presidente del Consejo Nacional de educación Juan B. Terán a cerrar las sesiones diciendo que "las Sociedades Populares reunidas en esta asamblea son la expresión más cabal de una conciencia educacional del país”21.

Rosario participó además de otros encuentros donde plasmó sus principios pedagógicos como el Primer Congreso Pedagógico realizado en el país en 1902, siendo encargada de la comisión de la revisión de planes de estudio y programas de las escuelas primarias y normales. Y en oportunidad de la celebración del primer centenario de la revolución de Mayo, concurrió al Primer Congreso Femenino Internacional que se celebró en Buenos Aires del 11 al 16 de mayo de 1910. El mismo que había sido convocado por la Asociación Universitaria Argentina distó de tener un tinte exclusivamente académico por el tono de sus debates y la participación y adhesión de mujeres no universitarias, argentinas y extranjeras, ligadas al mundo de la educación, gremial y político. Entre sus organizadoras se encontraban aquellas que llevaban más de una década de militancia y activismo en el Socialismo como las primeras médicas de Argentina - Cecilia Grierson, Julieta Lanteri, Elvira Rawson, Alicia Moreau

19 Carlos Vergara (1859-1929). Pedagogo mendocino se inscribe dentro de la corriente democráticoradicalizada fue partidario de la Escuela Nueva. Elvira Rawson (1864-1954) fue la segunda mujer en recibirse de médica en Argentina y Alicia Moreau (1885-1986) también médica, fueron integrantes del partido Socialista y activas defensoras de los derechos humanos y pacifistas.

20 Sandra Carli, rescató y analizó las Sociedades Populares de Educación en "Infancia y sociedad: la mediación de las asociaciones, centros y sociedades populares de educación" en Puigros Adriana, Sociedad civil y Estado en los orígenes del sistema educativo argentino, (Buenos Aires: Editorial Galerna, 1991).

21 José J. Berrutti, Las Sociedades Populares de Educación, en el centenario de la ley de educación común, (Sociedad Popular de Avellaneda, 1934). 
de Justo, Petrona Eyle - la odontóloga Sara Justo y las hermanas Chertkoff, Justa Burgos Meyer, Carolina Muzzilli, Juana María Begino y nuestra Rosario. La reunión, respondía a los siguientes objetivos:

1. Establecer lazos de unión entre todas las mujeres del mundo;

2. Vincular a las mujeres de todas las posiciones sociales a un pensamiento común; la educación e instrucción femeninas, la evolución de las ideas que fortifiquen su naturaleza, eleven su pensamiento y su voluntad en beneficio de la familia, para mejoramiento de la sociedad y perfección de la raza;

3. Modificar prejuicios, tratando de mejorar la situación social de muchas mujeres, exponiendo su pensamiento y su labor para poner de manifiesto las diversas fases de la actividad femenil y establecer las causales y efectos que determinan su influencia en el hogar, su condición de obrera, profesional, etc. y las soluciones de índole general y particular que tiendan a mejorar su situación.

En síntesis, se proponían debatir la situación y el rol de la mujer y abogar por la obtención de derechos sociales, políticos y civiles. En este sentido, "el Congreso apareció como la contracara de la Argentina moderna, donde la mayoría de sus habitantes estaba excluido del acceso a la ciudadanía política y las mujeres seguían siendo consideradas inferiores jurídicamente, al estar sujetas a la potestad masculina" 22 . En esa oportunidad, Rosario expuso una ponencia titulada "Acción concurrente de la mujer a la instrucción del pueblo: medidas que pueden ponerse en práctica"23.

En 1934 Rosario junto a otros importantes educadores participó de la Conferencia Nacional sobre Analfabetismo reunida en Buenos Aires con el objeto de conmemorar el cincuentenario de la sanción de la ley 1420. Eran años difíciles pues el nacionalismo impregnaba los contenidos, sin embargo hubo representantes de todas las provincias argentinas. En dicha reunión se consideraron entre otros temas los factores determinantes del analfabetismo, el plan para combatirlo y la acción de los particulares y del Estado, los medios para hacer cumplir la obligatoriedad de la educación, el analfabetismo de adultos en horarios especiales y la necesidad de actualizar las estadísticas de analfabetismo ${ }^{24}$.

22 Para ampliar la participación de las mujeres socialistas cfr. Lozano F Gil et al, Luchadoras. Historia de mijeres que bicieron historia, (Buenos Aires: Ediciones I.P.S, 2006).

23 Consejo Nacional de la Mujer, Primer Congreso Patriótico de Señoras en América del Sud, 1910. Antecedentes, sesiones, trabajos, juicios de la prensa, (Buenos Aires: Imprenta europea de M. A. Rosas. Ponencia de Rosario Vera Peñaloza), 128 a 139.

24 Para quien se interese en el tema cfr. Argentina. Ministerio de Justicia e Instrucción Pública, Conferencia Nacional sobre Analfabetismo, (Buenos Aires: octubre de 1934). 


\section{La Escuela Nueva y los jardines de infantes en la Argentina}

Hacia 1910 el positivismo iba perdiendo peso y era reemplazado por ideas espiritualistas y anti positivistas. Los uruguayos José Enrique Rodó, Vaz Ferreira, el mexicano Vasconcelos, el dominicano Pedro Henríquez Ureña, el peruano Alejandro Destua y el argentino Alejandro Korn, entre otros, fueron modificando el pensamiento de los dirigentes pues desde todos los sectores comienza a cuestionar el sistema político imperante. Poco después el fin de la Primera Guerra Mundial supuso importantes modificaciones en todos los órdenes, incluida la educación. Los conceptos plasmados a fines del XIX que se aplicaron primero en los países más adelantados del orbe, los cuales experimentaron cambios en diversos campos, sobre todo en el biológico, culminaron en ensayos educativos renovadores y en una reacción contra los sistemas vigentes.

La pedagogía pasó a ser una ciencia social y los estudios sobre la personalidad del niño, desde ese punto de vista, llevaron al convencimiento que la escuela no llenaba su finalidad social y que aquél era sometido a métodos que nada tenían que ver con su realidad. Así fue como nuevas teorías se expandieron y como era de esperar llegaron a la Argentina, aunque en un principio fueron tomadas con algún recelo. La reforma del pedagogo norteamericano John Dewey que se conoció aquí como Escuela Nueva fue aceptada por educadores progresistas, aunque implantarla llevó tiempo y adaptación mental, habida cuenta que con ella se revolucionaban las técnicas educativas como oposición a la escuela tradicional, que mantenía un rígido sistema verticalista y de aprestamiento del educando.

La Escuela Nueva era, en lo pedagógico, una reacción al positivismo, respondía a una visión paidocentrista de la enseñanza y retiraba el centro de la acción de las manos del docente y del sistema, para que fuese el alumno, específico consumidor de la enseñanza, quien tuviera mayor protagonismo y libertad. Los métodos basados en la experiencia, la observación y la percepción directa fueron utilizados por las nuevas teorías educativas. Entre los defensores de esta corriente se cuentan José Rezzano y su esposa Clotilde Guillén ${ }^{25}$. No estuvieron solos en la batalla, Juan P. Ramos, Juan E. Cassani, Juan Mantovani, entre otros, adhirieron a ese movimiento renovador que reconocía en el pedagogo italiano Ernesto Codignola una de las voces más eruditas del anti positivismo. Este

25 Rezzano desde la revista pedagógica La Obra adherida a la Liga Internacional de la Nueva Educación y, Guillén, desde la dirección de la Escuela Normal No 5 de la Capital Federal. María Vera de Flachs Cristina," "El reto al analfabetismo. 1920-1980", en La Educación en Córdoba. Siglo XX, capítulo V, (Córdoba: Ediciones del Copista, 1998), 105 a 154. 
grupo se propuso además dar al magisterio una mejor formación pedagógica que consistió en la reforma de los programas de pedagogía y didáctica de acuerdo a las nuevas direcciones del pensamiento pedagógico.

Rosario Vera Peñaloza fue otra de las impulsoras de esta corriente, a la vez que fue difusora de los principios de Fröebel y Montessori a quienes como señalamos estudió comparándolos y adaptándolos a la realidad $\operatorname{argentina~}^{26}$. Logró ensamblar la rigidez montessoriana con el excesivo simbolismo froebeliano; entre otras cosas recomendaba la observación de la naturaleza y el aprovechamiento de los variados e innumerables materiales que proporciona la misma como el algodón, paja, lana, piedras, arena etc., con los que las maestras podrían permitirse una mayor creatividad con bases científicas; a su criterio nada debía quedar librado a la improvisación.

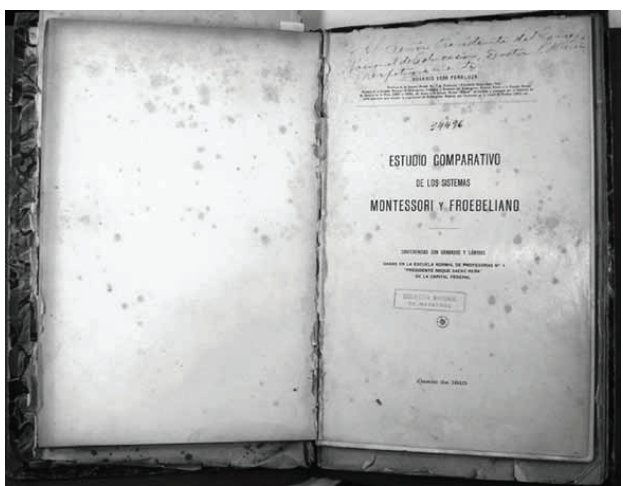

Foto 3. Estudio comparativo de los sistemas Montessori y Froebeliano de Rosario Vera Peñaloza. 1915.
La ley de educación $\mathrm{N}^{\circ} 1420$, sancionada el 8 de julio de 1884 , después de fuertes debates fue la piedra basal del sistema educativo nacional. La misma en su art. 11 había previsto lo siguiente: "Además de las escuelas comunes mencionadas, se establecerán las siguientes escuelas especiales de enseñanza primaria: uno o más jardines de infantes, en las ciudades donde sea posible dotarlos suficientemente..."27.

Sin embargo hasta las primeras décadas del siglo XX poco se hizo en beneficio de los más pequeños, a pesar de los esfuerzos de Sara Eccleston difusora de este nivel y fundadora de la Unión Froebeliana Argentina en 1893 desde donde divulgó el método froebeliano que encontró no pocos detractores entre los pedagogos positivistas ${ }^{28}$. Hubo que esperar a la época del peronismo para que la enseñanza pre escolar se incentivara.

26 Friedrich Wilhelm August Fröbel nació en Turingia el 21 de abril de 1772. En 1844 fundó un jardín de infantes que debió cerrar por falta de recursos. Escribió varias obras para dar a conocer su método sobre la naturaleza del niño, sus tendencias, inclinaciones y afecto. La educación, (Buenos Aires: 1886), "Breves apuntes sobre los jardines de infantes", 163.

27 Republica Argentina, Registro Nacional, Año 1884.

28 Se ocupó de este tema Sandra Carli "El campo de la niñez", en Historia de la Educación en la Argentina, T. III, Escuela, Democracia y Orden (1916-1943) Adriana Puigros Dir., (Buenos Aires: Editorial Galerna, 1993), 142 y 143. 


\section{Asociación Pro-difusión del Kindergarten}

Hacia 1905 Leopoldo Lugones, en ese momento Inspector de Enseñanza Secundaria y Normal, elevó a la Nación un informe adverso sobre los jardines de infantes ${ }^{29}$. Previamente él consultó a los directores de las escuelas normales acerca de la mentalidad y hábitos de los niños que habían asistido a los jardines de infantes y la respuesta fue que "eran inferiores en mentalidad y generalmente eran los rezagados y fracasados, sin contar los casos de indisciplina". Esto convenció al Ministro de Instrucción Pública, Joaquín V. González, de lo poco eficaz y perjudicial que podía resultar que lo niños iniciaran la escolaridad antes de los siete años; al tiempo que señaló que resultaban excesivamente costosos. Estas conclusiones tuvieron su correlato en el detenimiento del crecimiento del nivel, llegándose incluso a cerrar muchos jardines de infantes. Una de las voces que más resistieron esa medida fue la de Rosario Vera Peñaloza quien, en colaboración con otras colegas de brillante trayectoria, y como obedeciendo al mandato que recogieran de labios de su maestra Sara Chambelin de Eccleston, concibieron y fundaron la Asociación Pro Difusión del Kindergarten, cuya primera presidenta fue Rita Latallada de Victoria, una maestra kindergartiana egresada de la Escuela Normal de Paraná. Dicha institución reconocía como antecedente la Unión Froebeliana Argentina fundada por Sara de la que hicimos referencia.

La constitución de la nueva asociación tenía como primer propósito revertir la situación provocada por el informe adverso de Lugones e instalar el método frobeliano en el país en tanto el mismo había encontrado no pocos detractores entre los pedagogos positivistas. A este grupo de maestras pertenece el texto El kindergarten en la Argentina, didáctica froebeliana, en donde se perfila la planificación didáctica y la normativa vigente, en esa época, anterior a la organización de la formación de la maestra jardinera. Desde la sociedad se impartieron directivas para la formación de las nuevas maestras jardineras que demostraron verdadera vocación en esa especialidad, orientaron y crearon nuevos jardines de infantes, hasta conseguir que los cursos que se dictaban en ella fueran reconocidos oficialmente, lo que dio lugar a la fundación del Instituto "Mitre", que en la actualidad es la escuela formativa de maestras jardineras de mayor arraigo en Argentina.

Entretanto Eccleston tradujo El niño y la naturaleza, el manual de la baronesa Morenholtz Bulow, en el que Fröebel expuso sus doctrinas sobre

29 Leopoldo Lugones nació en Villa María de Río Seco, Córdoba, el 13 de junio de 1874. En su provincia inició estudios de periodismo. Viajó a Buenos Aires en 1896 donde se desempeñó como inspector de enseñanza y luego como director de la Biblioteca Nacional de Maestros. Trabajó como redactor del diario La Nación, por lo que viajó varias veces a Europa. Desde joven militó en el Partido Socialista y luego en el nacionalismo antiliberal, hecho que lo ubicó en el centro de las polémicas. Con sus obras enriqueció la prosa modernista. Se suicidó en febrero de 1939. 
la enseñanza, texto que llegó a ser de uso obligatorio por largos años en las escuelas normales ${ }^{30}$. Y se trasladó a Mendoza donde colaboró en la creación del jardín de infantes modelo dependiente de la escuela Normal y del cual fue su directora una antigua discípula suya Custodia Zuloaga. Por ese tiempo se abrieron varios otros jardines de infantes en Buenos Aires como el de el Jardín de Infantes Mitre (1924) y el del Jockey Club (1929). El socialismo luchó con denuedo para difundirlos en su afán de beneficiar a los sectores populares más postergados.

En la década del 30 y como consecuencia de la crisis económica varios sectores de la población se vieron vulnerados, particularmente los niños. Fue entonces cuando se observa un progresivo reconocimiento por parte del Estado de la necesidad de intervenir en la regulación de la economía apuntando al pleno empleo y favoreciendo un incremento de las políticas sociales efectuada por especialistas (sanitaristas, higienistas, trabajadores sociales, etc.) dirigidas a diversas áreas, entre ellas, la educación. Dichas tendencias, se corresponden con el contexto histórico mundial del Estado de Bienestar. Si bien dicha política no incidió para que los jardines de infantes se multiplicaran en el país sirvió para que el tema fuese ocupando un lugar en la agenda política argentina.

Con una marcada agudeza intelectual, Rosario en 1932 señalaba que las llamadas "escuelas nuevas" eran muy diversas entre si y que era muy compleja la tarea de marcar semejanzas o de establecer principios comunes. Por entonces, dictó una conferencia titulada "Los Jardines de Infantes y las Escuelas Nuevas". Allí polemizó con los escolanovistas Montessori y Decroly, y sostuvo que en los Jardines de Infantes fröebelianos es donde se sentaron las bases didácticas para renovar el trabajo áulico. En un tramo de la conferencia sostuvo que:

"Hay en la creación de Fröebel, una base lógica y un alcance que dan estabilidad a su sistema, haciéndolo servir de base para todas las reformas; de abi que las mismas escuelas nuevas, hayan echado mano de las ocupaciones Fröebelianas. Si observamos sus prácticas, siempre son a base de recortado o calado, doblado, modelado, cribado, trenzado, tejido, etc., los trabajos auxiliares de las iniciaciones y de todas las manualidades escolares, dentro de los ensayos de la escuela activa. (...) Es que dichas ocupaciones no fueron elegidas al caso por el gran pedagogo, ellas han sido el fruto de la observación de las tendencias y gustos del niño. La sistematización fue obra de los discipulos, como lo es actualmente, la corrección de este error que saca al trabajo manual de la rigidez de series, para presentarlo en forma de favorecer las iniciativas del niño"'31.

30 Bulow, B Marenholtz, El niño y su naturaleza. Exposición de las doctrinas de Fröebel sobre enseñanza, [traducido por Sara C. Eccleston] (Nueva York: D. Appleton y Cía, 1919).

31 Rosario Vera Peñaloza, "El Museo argentino para la Escuela primaria", en Boletín de la Dirección de Historia, letras y Ciencias, año IV, Homenaje a Rosario Vera Peñaloza (Córdoba: Mayo de 1980), 14 a 18. 
La creación en la Capital Federal del profesorado "Sara Ch. de Eccleston", el Jardín de Infantes "Mitre" (a modo de escuela de aplicación) y el Jardín de Infantes del Instituto Bernasconi donde Rosario dejó su impronta implicaron de algún modo el preludio para una política de expansión que se desplegaría en todo el país con más fuerza durante la siguiente década. Por ejemplo, en 1946 la Ley 5096 conocida como ley Simini, planteó para la provincia de Buenos Aires la obligatoriedad del jardín de infantes gratuito desde los tres hasta los cinco años ${ }^{32}$. Esta ley fue derogada en 1951 siendo reemplazada por otra (Ley 5650) que dispuso que el nivel preescolar era voluntario aunque creaba la Inspección General de los Jardines de Infantes, lo que jerarquizó dicho nivel. Simultáneamente el discurso oficial peronista basado en que "los únicos privilegiados son los niños", ayudó a fortalecer el sistema, pero eso es otra historia.

\section{Complejo Museológico del Instituto "Félix Bernasconi"}

Después del golpe militar de 1930 las tendencias educativas en Argentina se modificaron y muchas instituciones estuvieron sujetas a esos vaivenes políticos. Es en esta etapa cuando Rosario pudo concretar un viejo sueño que tuvo desde el inicio de su carrera. En 1931 a instancias del Consejo Nacional de Educación pudo crear el Primer Museo Argentino para la Escuela Primaria, que aún funciona en la Capital Federal, bajo el nombre de "Complejo Museológico" del Instituto Félix Bernasconi33. Dicho Museo según definición de su promotora era de carácter nacional y la idea del mismo había nacido al calor de las ideas del Dr. Joaquín V. González, un riojano que como ella insistía en la importancia de la geografía argentina como base de toda enseñanza lo que, fue bastante resistido por sus pares ${ }^{34}$. La geografía argentina sería la "bisagra"

32 Conocida como ley Simini en tanto fue impulsada por el diputado Jorge Simini, otorgó al Jardín de Infantes la jerarquía y el valor pedagógico que no se le había reconocido aún en nuestro sistema educativo. Este hecho colocó a la Argentina entre los primeros países del mundo en la materia. En el proyecto, el diputado expresó conceptos por siempre vigentes: "El Estado se ha olvidado del tramo del niño de 3 a 6 años. Y más sorprende el olvido, cuánto que es precisamente en esta edad, llamada preescolar, cuando mejor puede bucearse el campo físico, psíquico, de modo perfecto y con tiempo para prevenir con éxito, deficiencias y males que, descuidados, darán seres adultos que mucho costarán a la sociedad, por su escasa ó ninguna contribución al bien social, cuando no por resultar valores morales que sean negativos a ella. $\mathrm{Y}$, ¿qué se ha hecho por educar a nuestros pequeños? Muy poco de lo mucho de lo que corresponde hacer".

33 Félix F. Bernasconi, hijo de inmigrantes suizos nació en Buenos Aires en 1860. Dueño de una fortuna adquirió varias propiedades. En 1921 hizo construir un palacio en los altos de lo que fuera la ex quinta del perito Francisco P. Moreno. Al fallecer Bernasconi el edificio fue donado al Consejo Nacional de Educación con el único propósito de construir un "Palacio-Escuela" gratuito y obligatorio, para todos los niños humildes del Sur de la ciudad de Buenos Aires.

34 Rosario Vera Peñaloza, "El museo argentino para la escuela primaria. Plan General del Museo Argentino para la Escuela Primaria. Doctrina, alcance y forma", Rosario, 1929, en El Monitor de Educación Común, (diciembre de 1937), 99. Susana V García. "Museos y materiales de enseñanza en la Argentina 1890-1940" en El museo en escena, Politica y cultura en América Latina. Américo Castillo (Comp.), (Buenos Aires: Barcelona, México, Paidós, 2008). 
que relacionaría "científicamente" a todos los conocimientos humanos, para así "vigorizar el sentimiento de argentinidad". El Monitor de Educación Común publicó complicadísimos diagramas presentados por Vera Peñaloza, a los efectos de demostrar cómo desde la geografía argentina, podían desarrollarse los temas vinculados con las ciencias naturales, el hombre, su historia, su cultura, sus herramientas, en fin, todo, hasta llegar incluso a la aritmética ${ }^{35}$.

El vastísimo legado -más el trabajo infatigable y ad honorem a lo largo de 17 años de la fundadora- profundizó la importancia del Museo que en sus diferentes salas alberga las eras geológicas, restos fósiles, aspectos del suelo, alfarería, numismática, escudos provinciales, folklore argentino y un almacén de ramos generales y la pulpería "La surtida". Incluía además una sala de zoología, con invertebrados, vertebrados; hábitats, comunidades acuáticas, sala de educación para la salud, con los diferentes sistemas y sentidos del cuerpo humano y mineralogía. La idea era que el niño comprendiera el contenido de las ciencias sin la fatiga del enciclopedismo que constituyen tantas materias de estudios, a veces, presentadas sin conexión. Conociendo la región que habitan se debe hacer conocer el mundo que los rodea. Paralelamente, Rosario creó la cátedra de literatura infantil y de estudios folklóricos en la que los maestros aprendían a conocer y utilizar elementos del acervo nativo para mantener el carácter nacional en un país con tanta inmigración. Después de abandonar la dirección del Museo de su creación, esta maestra riojana prosiguió con su labor docente con más entusiasmo y dinamismo que nunca.

\section{Sus últimas realizaciones y deseos póstumos}

En 1949, pocos meses antes de su fallecimiento, instaló en el Instituto Sanmartiniano de la Capital Federal un mapa de su creación de América del Sud, realizado en relieve en el que se destacan las rutas seguidas por la expedición libertadora de San Martín a Chile y Perú. Dos o tres veces por semana concurría a dicho Instituto y personalmente explicaba a la concurrencia y delegaciones escolares que lo visitaban, la trayectoria seguida por los ejércitos, los lugares donde se libraron las batallas, como asimismo el pensamiento que orientó al Gran Capitán de América.

Su obra literaria y educativa dejada en libros y folletos fue fecunda y útil, aunque sus escritos hoy son poco recordados. Entre otros títulos mencionamos: "Credo Patriótico", "Vida del General San Martín", adaptada para los niños; "Seis rutas del paso de los Andes", "La Enseñanza de la geometría", "Enseñanza

35 Carlos Escude, El fracaso del proyecto argentino, (Buenos Aires: Ed. Tesis/Instituto Torcuato Di Tella, 1990). 
práctica de las fracciones", "Trabajo Manual", "Vulgarización científica, los jardines de infantes" y "Lectura del mapa de relieve de la República Argentina", etc. ${ }^{36}$.

Era tal su pasión por la enseñanza que, en 1950, con 77 años y mientras dictaba un curso en Chamical, La Rioja, encontró la muerte el 28 de mayo. Había dejado tras de sí sesenta y un años dedicados por entero a la educación infantil. En su homenaje, ese día se declaró "Día de los Jardines de Infantes". Como carecía de herederos forzosos y de bienes que no fuesen cosas insignificantes de su uso personal y su obra intelectual, nombró como albaceas a los Doctores Jorge y Ricardo Vera Vallejo y a su hija espiritual doña Martha Alcira Salotti a quien encomendó la revisión y ordenación de sus trabajos en materia docente, filosófica o literaria con el fin de publicarlos ${ }^{37}$. Renunció a sus derechos de autor en beneficio de la Junta de Historia y Letras de La Rioja, a la que ya le había ofrecido sus trabajos para que los publicaran. Y manifestó que si la edición produjera algún beneficio se destinará lo necesario para servir de base a la fundación de una escuela que llevará el nombre de su tía y madre de crianza doña Jesusa Peñaloza de Ocampo, en el pueblo de Malanzán donde había pasado su infancia. En dicha escuela se proporcionará a los niños la enseñanza necesaria para despertar vocaciones y desarrollar aptitudes latentes en cada uno, enseñando con el material que brindaba el medio ambiente.

\section{CONCLUSIÓN}

Desde los lejanos días de su juventud Rosario trasmitió sus saberes y desparramó sus conocimientos en escuelas de La Rioja, Paraná, Córdoba y Capital Federal. A través de su labor y de sus propios textos puede vislumbrarse como sus mayores empeños fueron puestos en los Jardines de Infantes a los que consideraba una institución educativa integral, útil, necesaria, imprescindible e insustituible. Con este convencimiento dio batalla por la instalación y la difusión de los mismos en cada rincón del país. Preocupada, como siempre se mostraba, por la suerte de éstos se dedicó a estudiar en profundidad la obra de Fröebel y de Montessori aunque también se ocupó de leer y analizar detenidamente la de los pedagogos del movimiento de Escuela Nueva, por lo que podemos inferir que sus ideas pedagógicas, en algún sentido, tienen puntos de contacto con Decroly, Montessori y las Agazzi, aunque sus concepciones tienen un núcleo fuertemente Fröebeliano.

\footnotetext{
Cfr. La bibliografía anexa.

Martha Salotti, su alumna y heredera, editó tras la muerte de Rosario doce trabajos científicos y el Instituto Sanmartiniano le confirió el Primer Premio por su "Credo Patriótico" y una condecoración por "Vida del General San Martín", adaptada para los niños.
} 
Cuando los precursores de la Escuela Nueva señalaron la naturaleza como escenario, al niño como actor, el interés como excitante, la observación y experimentación como vehículo de conocimiento, el trabajo como acción creadora; la libertad como sistema de disciplina y la responsabilidad como medio de gobernarse a sí mismos, no pensaron, acaso, en las dificultades que esos propósitos implicaban. Sin embargo, al lanzar tales ideas confiaron en que los maestros de verdad serían capaces de vencer tales obstáculos. Tales preocupaciones fueron las de Rosario Vera Peñaloza quien a lo largo de su dilatada existencia supo configurar una pedagogía de excepción poniendo énfasis en "el amor a la patria y en la perfección del individuo" partiendo de las grandes concepciones de Aristóteles, Pestalozzi y Federico Fröebel.

En síntesis, sus preocupaciones se centraron en cuatro áreas de reflexión: la reforma educativa; los Jardines de Infantes, la formación docente y la Pedagogía y la Didáctica dejando en todas ellas su impronta.

\section{FUENTES}

Consejo Nacional de la Mujer, Primer Congreso Patriótico de Señoras en América del Sud, 1910. Antecedentes, sesiones, trabajos, juicios de la prensa, (Buenos Aires: Imprenta europea de M. A. Rosas. Ponencia de Rosario Vera Peñaloza).

Entre Ríos, Revista del Consejo General de Educación, 200 años de Educación entrerriana. 2010. “El Eco de Córdoba”, periódico de Córdoba, 27 de mayo de 1869, № 1877.

El Popular, Olavarría, Provincia de Buenos Aires, 10 de agosto de 2012.

República Argentina, Ministerio de Justicia e Instrucción Pública, Primera Conferencia Nacional sobre Analfabetismo, reunida en Buenos Aires, octubre y noviembre de 1934, antecedentes, actas y conclusiones. Buenos Aires: Talleres Gráficos de la Penitenciaría Nacional, 1935.

Monitor de la Educación Común. No 502 - al 504 (1914) - Consejo Nacional de Educación, "Revista de revistas".

Vera Peñaloza, Rosario. La enseñanza práctica de la geometría en la escuela primaria, Ed. La Baskonia, Bs. As, 1900.

Vera Peñaloza, Rosario. "Asociación Nacional del Profesorado. La Asamblea en el Odeón". Revista El monitor de la educación común. Bs. As, 1912.

Vera Peñaloza, Rosario. Estudio comparativo de los sistemas Montessoriy Fröebeliano_Buenos Aires, Sociedad Nacional de Kindergarten, 1915.

Vera Peñaloza, Rosario. "Discurso en la Escuela Normal de Profesoras 1915". Revista El monitor de la educación común. Bs. As, 1915.

Vera Peñaloza, Rosario. "Enseñanza de la naturaleza", Revista El monitor de la educación común. Bs. As, 1916.

Vera Peñaloza, Rosario. Nuevas orientaciones en la educación de la mujer argentina, 1921.

Vera Peñaloza, Rosario. El museo argentino para la escuela primaria. Plan General del Museo Argentino para la Escuela Primaria. Doctrina, alcance y forma, 1929. 
Vera Peñaloza, Rosario. Los Jardines de Infantes y las Escuelas Nuevas, Sociedad de ex-alumnos de la Escuela Normal de Paraná, 1932.

Vera Peñaloza, Rosario, et al. El Kindergarten en la Argentina, Didáctica Froebeliana, Buenos Aires: Instituto Félix Bernasconi, 1936.

Vera Peñaloza, Rosario. La personalidad de Ernesto Bavio. Folleto publicado por el Diario la Nación, 1938.

Vera Peñaloza, Rosario. "Los artífice de la cultura nacional argentina", Revista El monitor de la educación común, (1940).

Vera Peñaloza, Rosario. "Discurso en Homenaje al Dr. Carlos Biedma", publicación de la Escuela Argentina Modelo, 1947.

Vera Peñaloza, Rosario. Prólogo del libro La lengua viva contribución experimental de Martha Salotti, 1950.

Vera Peñaloza, Rosario. Prólogo del libro Juguemos en el bosque de Martha Salotti, 1950.

Vera Peñaloza, Rosario. “Comentario del libro Mis montañas de Joaquín V. Gonzálež” Publicado por el Consejo Nacional de Educación, s/f.

Vera Peñaloza, Rosario. El paso de Los Andes por las seis rutas, Mendoza, Dirección General de Escuelas, Mendoza, 1936.

\section{REFERENCIAS}

Castillo, Américo (Comp.). El museo en escena, Politica y cultura en América Latina. Buenos Aires: Barcelona, México, Paidós, 2002.

Cossettini, Olga. La escuela viva. Buenos Aires: Losada, 1942.

Fernández Doux, Norma. "La Escuela de Paraná”. Y “El primer jardín de infantes del país”. Entre Rios, Revista del Consejo General de Educación, 200 años de Educación entrerriana, 2010.

Instituto Social de la Universidad Nacional del Litoral, Biblioteca pedagógica, Pensamiento Vivo de Rosario Vera Peñaloza, Universidad Nacional del Litoral, 1954.

Sarmiento Domingo F. "Discursos parlamentarios" en Obras completas, T. XX, vol., III, Belín Sarmiento Editor, La Facultad, 1914.

Vera de Flachs, María Cristina. "El Reto al analfabetismo. 1920-1980”, en La Educación en Córdoba. Siglo XX, capítulo V, Córdoba, Ediciones del Copista, 1998.

Vera de Flachs, María Cristina y Riquelme Norma. La educación primaria en Córdoba, 1930-1970. Crecimiento y Contradicciones, Junta Provincial de Historia de Córdoba, Córdoba, 1987.

Vera de Flachs, María Cristina, "Rosario Vera Peñaloza: maestra de la patria" en Educadores latinoamericanos, T. IV, Siglos XX y XXI. Soto Arango, Diana et. Tunja, Ediciones Doce Calles S.L., 2011.

Vera de Flachs, María Cristina "Rosario Vera Peñaloza una maestra que dejó huella en la historia de la educación de la Argentina". Revista Historia de la Educación Latinoamericana Vol. 14 No. 18, (2012): pp. 19 - 38. 